\title{
EDITORIAL
}

\section{Treg Cells to the Rescue}

\author{
David A. Fox
}

In this issue of Arthritis \& Rheumatism, Kong and colleagues report that collagen-induced arthritis (CIA), a mouse model of rheumatoid arthritis (RA), can be effectively treated by systemic injection of Treg cells (1). These results raise several questions: Are there defects in Treg cell numbers or function in RA? How should Treg cells be collected, generated, or expanded for use in the treatment of immune-mediated diseases? What are the risks of using Treg cells in human rheumatic diseases? Would such an approach be practical? And can we correct Treg cell dysfunction in vivo using other approaches that do not require direct infusion of these cells?

\section{Immune responses are regulated at multiple levels}

The adaptive immune response is mediated by $\mathrm{T}$ and $\mathrm{B}$ lymphocytes that possess clonal specificity for peptide or nonpeptide antigens, both foreign and self. Negative selection (death) of highly autoreactive T cells occurs during the development and maturation of these cells in the thymus, but the process is incomplete, and autoreactivity persists in the repertoire of $\mathrm{T}$ cells that are found in normal lymphoid organs and in the circulation. A system of checks and balances is needed not only to limit the potential for this persistent autoreactivity to evolve into autoimmune disease, but also to calibrate the degree and duration of immune responses to foreign antigens, so that successful host defense does not lead to excessive chronic inflammation. Table 1 lists some of the cells of the immune system that have roles in regulating $\mathrm{T}$ lymphocyte responses. Thus, Treg cells do not function in isolation, but instead in the context of (and often in concert with) other cells, such as Breg cells (2), Ann Arbor.

David A. Fox, MD: University of Michigan Health System,

Address correspondence to David A. Fox, MD, University of Michigan Health System, Room 3918 Taubman Center, 1500 East Medical Center Drive, Ann Arbor, MI 48109-0358. E-mail: dfox@umich.edu.

Submitted for publication March 23, 2012; accepted in revised form April 17, 2012. dendritic cells, natural killer $\mathrm{T}$ cells, $\mathrm{CD} 8+\mathrm{T}$ cells (known long ago as suppressor $\mathrm{T}$ cells), and others.

\section{Characteristics of Treg cells}

Although immunoregulatory roles of CD4+ $\mathrm{T}$ cells had been described in the 1980s, the recognition in 1995 of CD25 (a subunit of the interleukin-2 receptor [IL-2R]) as a surface marker of such cells allowed Treg cells to be more specifically identified and studied (for review, see ref. 3). The subsequent discovery of a prototypic transcription factor that is expressed in Treg cells, FoxP3, was a key advance not only in identifying Treg cells, but also in understanding their function. FoxP3 shapes the pattern of gene expression in Treg cells, and deficiency of this protein in mice or humans leads to life-threatening autoimmunity (for review, see ref. 3). Nevertheless, FoxP3 expression is not always an accurate indicator that a cell is a Treg cell, as FoxP3 can be briefly expressed during the activation of other $\mathrm{T}$ cells and Treg cells can occasionally lose FoxP3 expression, at least transiently, while maintaining Treg cell function (4).

Cells of the immune system can typically be subdivided into two or more subsets, and Treg cells are no exception - they can be subclassified as either natural Treg (nTreg) cells or induced Treg (iTreg) cells. Natural Treg cells arise in the thymus and exist in the mature $\mathrm{T}$ cell population prior to stimulation by encounter with antigen. Induced Treg cells arise, on the other hand, as a result of antigenic stimulation in the mature immune system. Both types of Treg cells express CD25 and can be expanded by IL-2. A recent report suggests that nTreg cells and iTreg cells may have unique, distinct, and nonredundant functional roles in the immune response (5). A further level of complexity has been created by the identification of an additional subset of iTreg cells, termed iTr35 cells, that secrete IL-35. IL-35 not only mediates some of the regulatory effects of these cells, but also induces non-Treg cells to differentiate into iTr35 cells (6). 
Table 1. Cells that regulate $\mathrm{T}$ cell responses

Treg cells (a subset of CD4+ T cells)

Dendritic cells

Natural killer T cells

Breg cells

CD8 + T cells

Effector Th cell subsets (e.g., Th1, Th2)

Treg cells carry out their regulatory functions both by secretion of cytokines, especially IL-10, and by direct cell-cell contact, in which CTLA-4 on the Treg cell surface is an important participant. Targets of Treg cells that are relevant to RA include other $\mathrm{T}$ cells, B cells, dendritic cells, and even osteoclasts (7). Direct Treg cell interactions with effector T cells can lead to the suppression of effector $\mathrm{T}$ cell proliferation and/or suppression of cytokine production. The complexity of Treg cell interactions with the entire immune system, including their ability to recruit other cells (especially dendritic cells) to function as secondary or surrogate mediators of cascades of immune regulation, means that assessment of Treg cell function requires multiple assays with diverse end points. Simple enumeration of these cells is not sufficient, and is ideally supplemented by a panel of functional studies.

\section{Treg cells in RA}

Treg cells are present in RA, and are not numerically deficient, either systemically or in inflamed synovium. However, they do not appear to function normally, and in some studies have failed to suppress cytokine production by effector $\mathrm{T}$ cells while still suppressing $\mathrm{T}$ cell proliferation (for review, see ref. 3). Multiple mechanisms may interfere with Treg cells in RA. The observation that tumor necrosis factor (TNF) can impair Treg cell function has been supplemented by the demonstration that anti-TNF treatment of RA restores defective Treg cell function to normal levels (8). Other cytokines, such as IL-6, can also interfere with Treg cell differentiation and function, and it is plausible that the antiIL-6R antibody tocilizumab also corrects defective Treg cell activity as one of its therapeutic mechanisms in RA. Beneficial effects of adoptive transfer of CD4+CD25+ $\mathrm{T}$ cells have been seen in animal models of RA, but a greater understanding of such an approach is needed before considering its use in human autoimmune diseases.

Consideration of the possible role of Th17 cells in RA prompts at least two reasons for concern that Treg cell infusions could actually make RA worse. (Th17 cells, which secrete IL-17 and were first recognized in 2005 $[9,10]$, have been implicated as important in RA and many other inflammatory immune conditions.) The first concern arises from the complex regulatory relationships that exist between the effector $\mathrm{T}$ cell subsets. The major cytokine products of Th1 cells (interferon- $\gamma$ ) and Th2 cells (IL-4) are potent suppressors of the development and function of Th17 cells, both in normal immune responses and in autoimmune arthritis models. Th1 and Th2 cell proliferation and cytokine secretion are, however, suppressed by Treg cells, while direct suppression of Th17 cells by Treg cells has been more difficult to document. Thus, a potential scenario is that Treg cell infusions, by suppressing Th1 and Th2 populations, would remove restraints on Th17 cells and make Th17related diseases worse.

A second concern arises from the recently recognized plasticity of CD4 $+\mathrm{T}$ cell subsets, which allows interconversion of differentiated subsets depending on the in vitro or in vivo cytokine milieu. The developmental pathway of Treg cells requires transforming growth factor $\beta$ (TGF $\beta$ ), while Th17 cell differentiation (in mice) requires TGF $\beta+$ IL-6. The close relationship between these pathways raises the possibility that in an IL-6-rich environment infused Treg cells could actually change into Th17 cells, while the development of new Treg cells would be suppressed. Moreover, a peptide containing critical residues of the RA-associated major histocompatibility complex allele that is termed the shared epitope, or a tetrameric form of the shared epitope, can cause dendritic cells to suppress Treg cell development and favor Th17 cell differentiation (11).

\section{Induced Treg cells to the rescue}

In this context, the report by Kong et al makes several important points. This group compared nTreg cells and iTreg cells in the treatment of established CIA. The nTreg cells were expanded from the mouse thymus by culture in medium that contained a type II collagen peptide and IL-2. The iTreg cells were generated from mouse spleen cells by culture with collagen peptide, IL-2, and TGF $\beta$. In the presence of IL- 6 , only the iTreg cells, which did not express IL-6R or the associated signaling molecule STAT-3, were able to sustain the ability to suppress effector $\mathrm{T}$ cell proliferation and cytokine production in vitro, and, importantly, they suppressed the development and function of Th17 cells. While either type of Treg cell could suppress CIA when injected into mice at the time of initial immunization with type II collagen, only the iTreg cells were able to 
attenuate CIA when the cells were administered 2 weeks after collagen immunization, which is $1-2$ weeks before arthritis develops in this model. Even after the appearance of clinical arthritis, iTreg cells, but not nTreg cells, suppressed further progression of disease. By labeling the infused Treg cells with a fluorescent dye, Kong et al followed their fate in vivo after injection of these cells into mice. Some of the nTreg cells died in the mice, while many lost expression of FoxP3 and converted to Th17 cells. In contrast, the iTreg cells survived, retained expression of FoxP3, and continued to function in the mice with CIA despite the in vivo abundance of proinflammatory cytokines. The iTreg cells even led to an accumulation of endogenous Treg cells in the lymph nodes in place of Th17 cells, possibly by conversion of Th17 cells to Treg cells.

This demonstration of a potent effect on a Th17driven autoimmune disease in vivo is an important step forward in demonstrating the encouraging therapeutic potential of iTreg cells. Whether the iTreg cells act directly on Th17 cells in vivo in CIA or through intermediates such as dendritic cells is presently unclear, and will be an interesting issue for further experimentation. Also unknown is whether late in disease Th17 cells can become resistant to regulation by iTreg cells, analogous to their acquisition of resistance to IL-4 $(12,13)$. The ultimate duration of the in vivo effects of iTreg cells remains to be clarified.

Nevertheless, the data reported by Kong et al provide impetus to the concept of enhancing Treg cell function as a strategy to treat human disease. Enhancement of Treg cell function might ultimately be accomplished by approaches that are simpler, safer, and less costly than infusion of in vitro-expanded iTreg cells. Diet can even influence the number of circulating Treg cells. In NZB $\times$ NZW mice, which are used as a model of systemic lupus erythematosus, caloric restriction (a maneuver known to retard the progression of lupus in these animals) lowers leptin levels and concurrently increases the number of Treg cells, an effect that is reversed by leptin replacement (14). The diverse nature of the factors that influence Treg cell number and function suggests that in future clinical trials of a wide variety of treatments for autoimmune diseases, Treg cell assays should be considered as worthwhile mechanistic studies. Such studies will lead to a deeper understanding of how these cells behave in human rheumatic diseases and in response to immunomodulatory therapies. The exciting goal of restoring defective immune regulation rather than resorting to immunosuppression as the primary approach to treating RA and other conditions will then be closer to reality.

\section{AUTHOR CONTRIBUTIONS}

Dr. Fox drafted the article, revised it critically for important intellectual content, and approved the final version to be published.

\section{REFERENCES}

1. Kong N, Lan Q, Chen M, Wang J, Shi W, Horwitz DA, et. al. Antigen-specific transforming growth factor $\beta$-induced Treg cells, but not natural Treg cells, ameliorate autoimmune arthritis in mice by shifting the Th17/Treg cell balance from Th17 predominance to Treg cell predominance. Arthritis Rheum 2012;64:2548-58.

2. Klinker MW, Lundy SK. Multiple mechanisms of immune suppression by B lymphocytes. Mol Med 2012;18:123-37.

3. Sarkar S, Fox DA. Regulatory $\mathrm{T}$ cell defects in rheumatoid arthritis [editorial]. Arthritis Rheum 2007;56:710-3.

4. Miyao T, Floess S, Setoguchi R, Luche H, Fehling HJ, Waldmann $\mathrm{H}$, et al. Plasticity of Foxp $3^{+} \mathrm{T}$ cells reflects promiscuous Foxp3 expression in conventional $\mathrm{T}$ cells but not reprogramming of regulatory T cells. Immunity 2012;36:262-75.

5. Josefowicz SZ, Niec RE, Kim HY, Treuting P, Chinen T, Zheng Y, et al. Extrathymically generated regulatory $\mathrm{T}$ cells control mucosal $\mathrm{T}_{\mathrm{H}} 2$ inflammation. Nature 2012;482:395-9.

6. Collison LW, Delgoffe GM, Guy CS, Vignali KM, Chaturvedi V, Fairweather D, et al. The composition and signaling of the IL-35 receptor are unconventional. Nat Immunol 2012;13:290-9.

7. Zaiss MM, Frey B, Hess A, Zwerina J, Luther J, Nimmerjahn F, et al. Regulatory $\mathrm{T}$ cells protect from local and systemic bone destruction in arthritis. J Immunol 2010;184:7238-46.

8. Nadkarni S, Mauri C, Ehrenstein MR. Anti-TNF- $\alpha$ therapy induces a distinct regulatory $\mathrm{T}$ cell population in patients with rheumatoid arthritis via TGF- $\beta$. J Exp Med 2007;204:33-9.

9. Harrington LE, Hatton RD, Mangan PR, Turner H, Murphy TL, Murphy KM, et al. Interleukin 17-producing CD4+ effector T cells develop via a lineage distinct from the T helper type 1 and 2 lineages. Nat Immunol 2005;11:1123-32.

10. Park H, Li Z, Yang XO, Chang SH, Nurieva R, Wang YH, et al. A distinct lineage of $\mathrm{CD} 4 \mathrm{~T}$ cells regulates tissue inflammation by producing interleukin 17. Nat Immunol 2005;11:1133-41.

11. De Almeida DE, Ling S, Pi X, Hartmann-Scruggs AM, Pumpens $\mathrm{P}$, Holoshitz J. Immune dysregulation by the rheumatoid arthritis shared epitope. J Immunol 2010;185:1927-34.

12. Sarkar S, Tesmer LA, Hindnavis V, Endres JL, Fox DA. Interleukin17 as a molecular target in immune-mediated arthritis: immunoregulatory properties of genetically modified murine dendritic cells that secrete interleukin-4. Arthritis Rheum 2007;56:89-100.

13. Cooney LA, Towery K, Endres J, Fox DA. Sensitivity and resistance to regulation by IL-4 during Th17 maturation. J Immunol 2011;187:4440-50.

14. Liu Y, Yu Y, Matarese G, La Cava A. Fasting-induced hypoleptinemia expands functional regulatory $\mathrm{T}$ cells in systemic lupus erythematosus. J Immunol 2012;188:2070-3. 